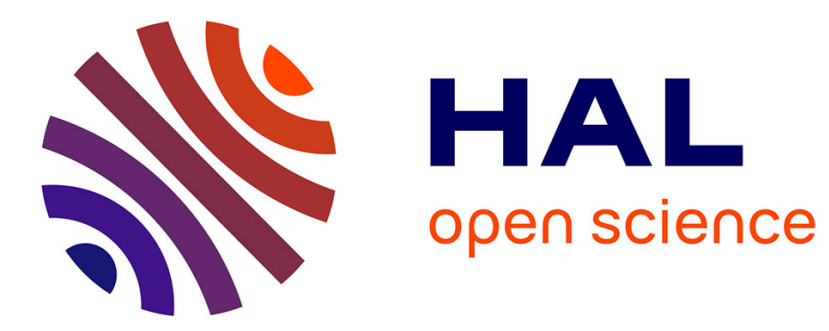

\title{
Sediment load determines the shape of rivers
}

Predrag Popović, Olivier Devauchelle, Anaïs Abramian, Eric Lajeunesse

\section{To cite this version:}

Predrag Popović, Olivier Devauchelle, Anaïs Abramian, Eric Lajeunesse. Sediment load determines the shape of rivers. Proceedings of the National Academy of Sciences of the United States of America, 2021, 118 (49), pp.e2111215118. 10.1073/pnas.2111215118 . hal-03497876

\section{HAL Id: hal-03497876 https://hal.science/hal-03497876}

Submitted on 20 Dec 2021

HAL is a multi-disciplinary open access archive for the deposit and dissemination of scientific research documents, whether they are published or not. The documents may come from teaching and research institutions in France or abroad, or from public or private research centers.
L'archive ouverte pluridisciplinaire HAL, est destinée au dépôt et à la diffusion de documents scientifiques de niveau recherche, publiés ou non, émanant des établissements d'enseignement et de recherche français ou étrangers, des laboratoires publics ou privés. 


\title{
Sediment load determines the shape of rivers
}

\author{
Predrag Popović $^{\mathrm{a}}$, Olivier Devauchelle ${ }^{\mathrm{a}}$, Anaïs Abramian ${ }^{\mathrm{b}}$, and Eric Lajeunesse ${ }^{\mathrm{a}}$ \\ a Université de Paris, Institut de Physique du Globe de Paris, 1 rue Jussieu, CNRS, F-75005 Paris, France; borbonne Université, CNRS - UMR 7190, Institut Jean Le Rond \\ d'Alembert, F-75005 Paris, France
}

This manuscript was compiled on November 16, 2021

\begin{abstract}
Understanding how rivers adjust to the sediment load they carry is critical to predicting the evolution of landscapes. Presently, however, no physically based model reliably captures the dependence of basic river properties, such as its shape or slope, on the discharge of sediment, even in the simple case of laboratory rivers. Here, we show how the balance between fluid stress and gravity acting on the sediment grains, along with cross-stream diffusion of sediment, determines the shape and sediment flux profile of laminar laboratory rivers which carry sediment as bedload. Using this model, which reliably reproduces the experiments without any tuning, we confirm the hypothesis, originally proposed by Parker (1), that rivers are restricted to exist close to the threshold of sediment motion (within about $20 \%$ ). This limit is set by the fluid-sediment interaction and is independent of the water and sediment load carried by the river. Thus, as the total sediment discharge increases, the intensity of sediment flux (sediment discharge per unit width) in a river saturates, and the river can only transport more sediment by widening. In this large discharge regime, the cross-stream diffusion of momentum in the flow permits sediment transport. Conversely, in the weak transport regime, the transported sediment concentrates around the river center without significantly altering the river shape. If this theory holds for natural rivers, the aspect ratio of a river could become a proxy for sediment discharge - a quantity notoriously difficult to measure in the field.
\end{abstract}

rivers | sediment transport | momentum diffusion | Poisson equation

$\mathbf{F}$ lowing from mountains to oceans, rivers traverse immense distances across the land, eroding, transporting, and depositing sediment along the way, thereby shaping much of the landscape we see on Earth (2-5). However, a precise understanding of how rivers adjust their shape to the amounts of sediment and water they transport is lacking. This is partly due to the difficulty of collecting sediment flux measurements in the field, and partly due to the complicated coupling between the flow and the sediment bed.

In rivers that carry a small amount of sediment, sediment grains are typically close to their threshold of motion - below this threshold, any sediment carried by a river would be deposited, building the river bed until it eventually reaches the threshold, while, above the threshold, uncompensated erosion of the bed would quickly bring the river back to the threshold (6). For this reason, early theories were formulated for inert rivers (rivers that do not transport sediment) and assumed that such rivers construct their own bed so that the grains on the bed surface are exactly at the threshold of motion (7-10). Under this assumption, they showed that the shape of the river channel is independent of its water discharge, which can only affect the size of the river. This threshold theory accounts for the observation that the width of rivers increases as the square root of their discharge, an empirical correlation known as Lacey's law $(10,11)$.

In active rivers (those that transport sediment), sediment transport is driven only by a small departure of the shear stress from its threshold value $(5,9)$. The minuteness of this departure makes the study of active rivers challenging. It means that, to find the sediment flux, one needs to measure or calculate the stress with high precision - simple order-ofmagnitude estimates are not sufficient (12). This is a daunting task, since the stress sensitively depends on the river shape, which, in turn, adjusts to the stress distribution.

Parker (1) first addressed the question of active rivers with a model in which a turbulent river splits into inert banks and a flat, active bottom. He found that the cross-stream diffusion of momentum, which distributes stress from faster flowing regions to slower ones, is essential to enable sediment transport in a stable river channel. His model qualitatively agreed with real rivers - he found that the stress on the river bed is at most about $20 \%$ above critical, which limits the intensity of sediment transport. It is, however, unclear why a river should sharply split into inert banks and a flat bottom, as required by Parker's model. Moreover, it is unclear how a river transitions from an inert, threshold channel to a singular configuration of Parker as its sediment discharge increases.

Since field measurements are difficult, a good place to test our understanding of rivers is the laboratory (13). However, even laboratory investigations have been a challenge in themselves (14-16) - stable single-thread rivers were only recently produced in a laboratory setting (17-19). Nevertheless, these experiments have been enlightening - by focusing on straight, laminar, stationary rivers, they presented strong support for the threshold hypothesis of inert rivers. So far, however, they have not been compared to Parker's theory for active rivers.

Another key insight that arose from experiments is that the grains that are carried as bedload (i.e. that are dragged along

\section{Significance Statement}

Rivers carry and deposit sediment, thereby shaping most landscapes around us. In doing so, their malleable channels change shape to accommodate the sediment load. Here, we show how fluid stress, gravity, and the erratic trajectories of traveling grains combine together to determine the shape of a river. We find that the stress on the bed of a river cannot be significantly above the critical value for sediment motion, which bounds the intensity of sediment transport and, thus, forces the river to widen as its sediment load increases. Although our results relate directly only to experimental, laminar rivers, they likely also apply qualitatively to natural ones, potentially allowing us to use the shape of a river as a proxy for its sediment discharge.

Authors declare no conflict of interest.

${ }^{2}$ All correspondence should be addressed to Predrag Popović. E-mail: arpedjo@gmail.com 
the river bed) (20) diffuse laterally by randomly hitting other grains on the bed surface along their way $(21,22)$. In analogy with a gas placed in a gravitational field, the balance between gravity and diffusion distributes the transported grains over the bed so that the concentration of moving grains exponentially falls off with increasing elevation above the channel centerline (22). This Boltzmann distribution of the moving grains relates the sediment flux to the shape of the river. The role of sediment diffusion was recognized early in rivers that transport their sediment in suspension (23), but these experiments have shown that this mechanism also applies to bedload transport.

In this paper, we use the experiments of Abramian et al. (19) (section 1) to understand what sets the channel shape of active rivers. In our theory, the above mechanisms combine to shape the river - the shape of the channel determines the stress, the stress determines the sediment flux, while the Boltzmann distribution relates the sediment flux back to the shape (section 2). In equilibrium, these mechanisms are all coupled together, and their simultaneous coexistence determines a unique river channel for given discharges of water and sediment (assuming the channel is straight and single-thread). Therefore, the problem can be solved self-consistently, at least in principle. However, this problem is difficult since the fluid stress anywhere on the bed depends on the entire shape of the river.

We bypass this issue by simplifying the equation for the fluid stress, assuming that the aspect ratio of a river (ratio of width to depth) is large (section 3). We then formulate a model for the steady-state shape of a straight, laminar river with bedload transport by using this minimal representation for the stress, and including the Boltzmann distribution for the moving grain density. This model takes the form of a second-order boundary value problem (BVP) which can be analyzed numerically (section 4) and analytically (section 5). We note that this is a well defined problem only for a river in equilibrium (steady-state), so that it does not answer how the river reaches this equilibrium.

In the limit of large water and sediment discharge, the river in our model splits into inert banks and a flat active bottom, exactly as prescribed in Parker's (1) model. We, thus, show how Parker's (1) model arises as a limit of our theory. We call this limit the "Parker regime" (section 6), and we define a condition for reaching it. Like Parker (1), we find that laminar rivers cannot exist far from the threshold of sediment motion and, thus, cannot accommodate a sediment flux (discharge per unit width) larger than a maximum. We find that this maximum depends only on the friction coefficient of the sediment, $\mu_{t}$. Since the sediment flux is bounded, a river in the Parker regime has to widen to accommodate a larger sediment discharge. Moreover, we find that momentum diffusion in the flow plays a key role in sediment transport. We compare our results with laboratory experiments and find good agreement without any tuning. In this way, for the first time, we provide support for Parker's hypothesis in a controlled setting. However, we also discover another qualitatively different regime, which applies to rivers with large water but small sediment discharge. In this "weak transport regime" (section 7), sediment transport relies on the diffusion of sediment, and a higher load is accommodated by increasing the sediment flux without altering the shape of the river which carries it. (a)

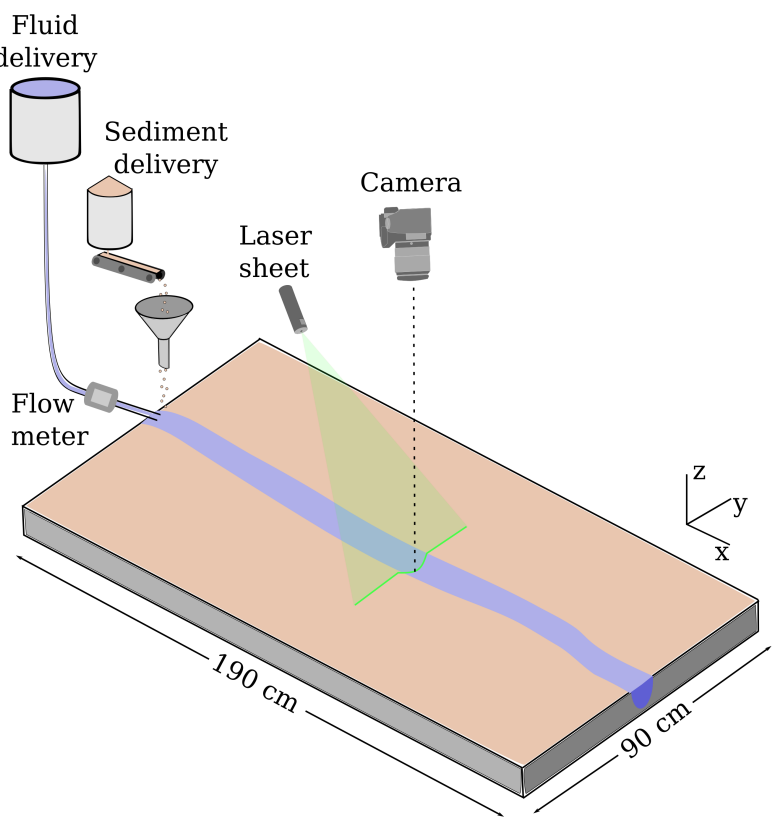

(b)

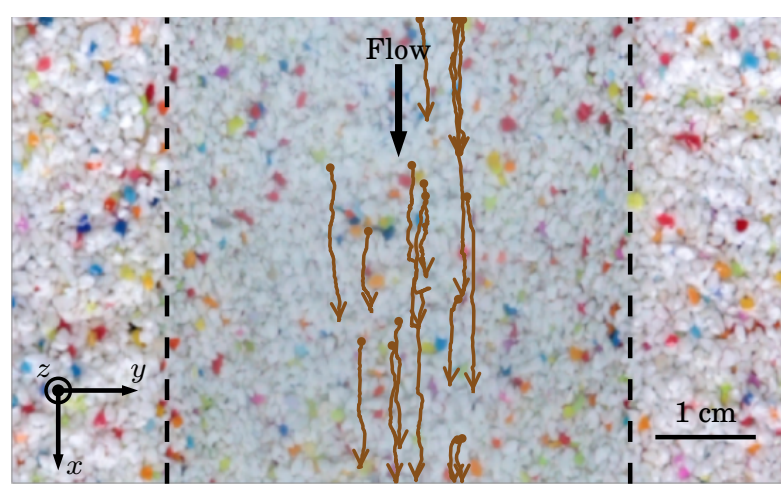

Fig. 1. (a) Experimental setup of Abramian et al. (19). (b) Photograph of the sediment bed taken with the overhead camera. Brown lines represent trajectories of tracked grains.

In both theory and experiments, we find that the aspect ratio of a river strongly depends on its sediment discharge. This suggests that, in the field, the shape of the river could be used as a proxy for its sediment load. To verify this, however, our theory would have to be adapted for turbulent flows - a task we leave for the future.

We also leave the mathematical details, tables for experimental runs, and other results that are not necessary to understand the main points of the paper to the Supplementary Information (SI).

\section{Experiments}

In this section, we briefly describe the experiments of Abramian et al. (19), which inspired the present theory. A schematic and a photograph of the experiment are shown in Fig. 1, and experimental parameters are summarized in Table S1 of the SI.

The setup consisted of an inclined tank, $190 \mathrm{~cm} \times 90 \mathrm{~cm} \times$ $10 \mathrm{~cm}$ in size, filled with plastic sediment made up of grains of diameter $d_{s}=0.83 \pm 0.2 \mathrm{~mm}$ and density $\rho_{s}=1490 \mathrm{~kg} \mathrm{~m}^{-3}$. At the inlet, a mixture of water and glycerol was pumped 


$$
Q_{s}=0 \text { grains } \mathrm{s}^{-1}
$$
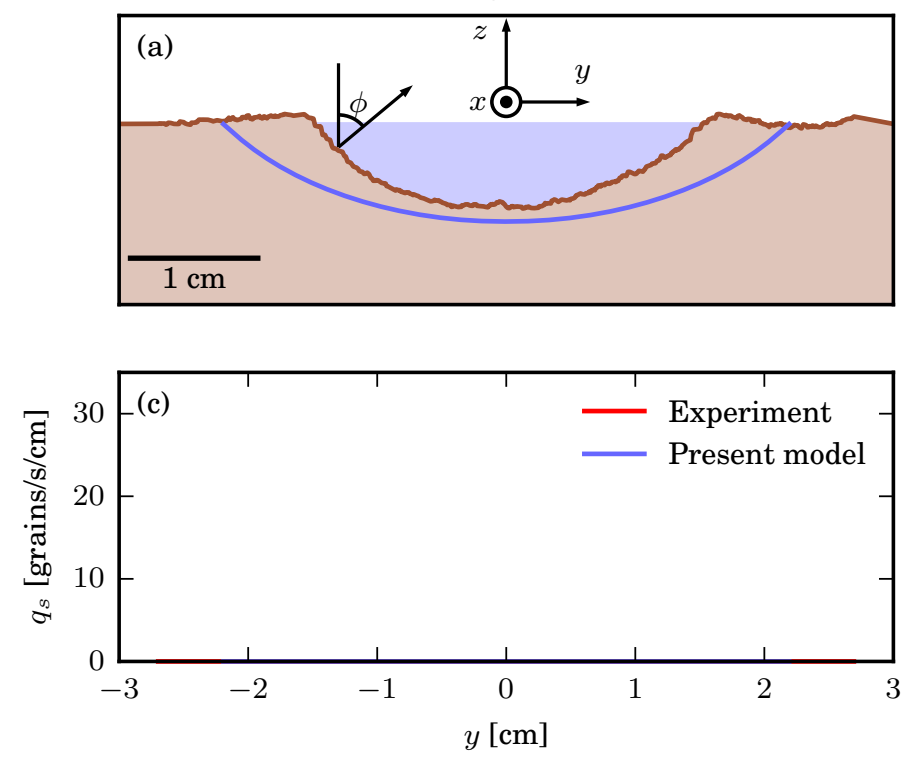

$Q_{s}=44$ grains s $^{-1}$
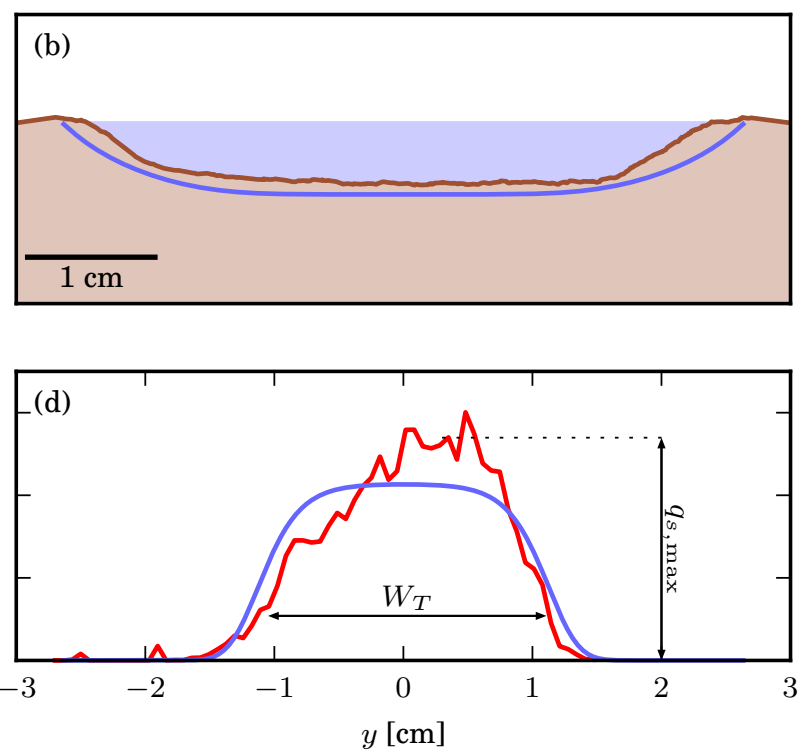

Fig. 2. (a) and (b) River cross-sections from the experiments of Abramian et al. (19) (brown line) and the present model (blue lines). Aspect ratio is preserved. (c) and (d) Corresponding sediment flux profiles, $q_{s}(y)$, for the experiments (red lines) and our model (blue lines). Panels on the left ((a) and (c)) correspond to an inert river (no sediment discharge, $Q_{s}=0$ ), while the right panels ((b) and (d)) correspond to an active one (sediment discharge $Q_{s} \approx 44$ grains s ${ }^{-1}$ ). The transport width, $W_{T}=Q_{s} /\left\langle q_{s}\right\rangle$, with $\left\langle q_{s}\right\rangle$ given by Eq. 1, as well as the maximum sediment flux, $q_{s, \text { max }}$, are marked with arrows in panel (d). The downstream slope, $S$, could not be measured accurately, but it is approximately $S \approx 0.005$ for the inert river and $S \approx 0.01$ for the active one.

into the tank, at a discharge $Q_{w} \approx 1 \mathrm{l} \mathrm{min}^{-1}$, which was kept as constant as possible during all experimental runs. The density and viscosity of the fluid were $\rho_{f}=1160 \pm 5 \mathrm{~kg} \mathrm{~m}^{-3}$ and $\nu=10^{-5} \mathrm{~m}^{2} \mathrm{~s}^{-1}$. The high fluid viscosity, achieved by adding glycerol to the mixture, kept the fluid flow laminar (the Reynolds number remained below about 10 in all experiments). The fluid found its way to the outlet at the opposite end of the tank, meanwhile carving its own channel through the sediment. Additionally, dry sediment was injected into the system at a prescribed rate, $Q_{s}$. Abramian et al. (19) performed 5 experimental runs in which they varied the sediment discharge between 0 and 60 grains s ${ }^{-1}$.

A typical river forms as follows. First, the experiment goes through a transient during which the fluid erodes more sediment than is injected at the inlet. At this stage, a single channel of width $W \sim 5 \mathrm{~cm}$ quickly forms, whose downstream slope, $S$, slowly changes over time until it reaches steady-state at $S \sim 0.01$. The duration of this transient, $T$, roughly corresponds to the time to build a sediment channel of constant slope, $S$, and width, $W$, over the entire length of the tank, $\mathcal{L} \sim 2 \mathrm{~m}$, by exchanging sediment at a rate $Q_{s} \sim 100$ grains s$^{-1}$ with the bed. A simple scaling analysis yields $T \sim \mathcal{L}^{2} W S /\left(d_{s}^{3} Q_{s}\right) \sim 5 \mathrm{~h}$, consistent with typical transients in the experiments. The exact duration of the transient depends on the initial setup of the experiment and can be shortened by, for example, setting the initial inclination of the tank close to the steady-state slope of the river. After reaching steady-state, the river transports as much sediment along its bed as is delivered by the sediment feeder. Sediment travels as bedload - grains roll, slip, and bounce on the sediment bed. The river channel typically appears to be roughly straight with only minor sinuosity, and, once formed, it does not move significantly. Moreover, the steady-state river is insensitive to the initial setup of the experiment - it selects its own width, $W$, depth, $D_{\max }$, and downstream slope, $S$, regardless of the initial conditions. Beyond a certain value of sediment discharge (about $Q_{s} \approx 90$ grains s $^{-1}$ ), the channel destabilizes into intertwined threads that form a braided river. The range of $Q_{s}$ explored in these experiments covered the entire range of sediment discharge for which a stable single-thread river can form.

To characterize the shape of these experimental rivers, Abramian et al. (19) measured the sediment bed elevation along a cross-section with a laser sheet. They constantly monitored the river using an overhead camera, and tracked the trajectories of moving colored grains, which allowed them to measure the profile of sediment flux, $q_{s}$, across the river (to avoid possible confusion, we emphasize here that the sediment discharge, $Q_{s}$, is the integral of the sediment flux, $q_{s}$, over the cross-section of the river). We show two rivers and their sediment flux profiles in Fig. 2; profiles for the other runs are shown in Fig. S1 and their properties are summarized in Table S2 of the SI. Most sediment concentrates near the channel center over a well-defined bed section of width $W_{T}$. We define this transport width, $W_{T}$, as the width that relates the sediment discharge and the mean sediment flux, $Q_{s}=W_{T}\left\langle q_{s}\right\rangle$. To make $W_{T}$ a robust quantity resistant to experimental noise, we define $\left\langle q_{s}\right\rangle$ to be the average sediment flux over a probability density function $q_{s} / Q_{s}$, so that

$$
\left\langle q_{s}\right\rangle \equiv \frac{1}{Q_{s}} \int_{-W / 2}^{W / 2} q_{s}^{2}(y) \mathrm{d} y .
$$

Figure 3 and Fig. S2 of the SI illustrate how the characteristics of laboratory rivers change as the sediment discharge, $Q_{s}$, increases: the rivers become wider, shallower, steeper, and transport sediment more intensely. 

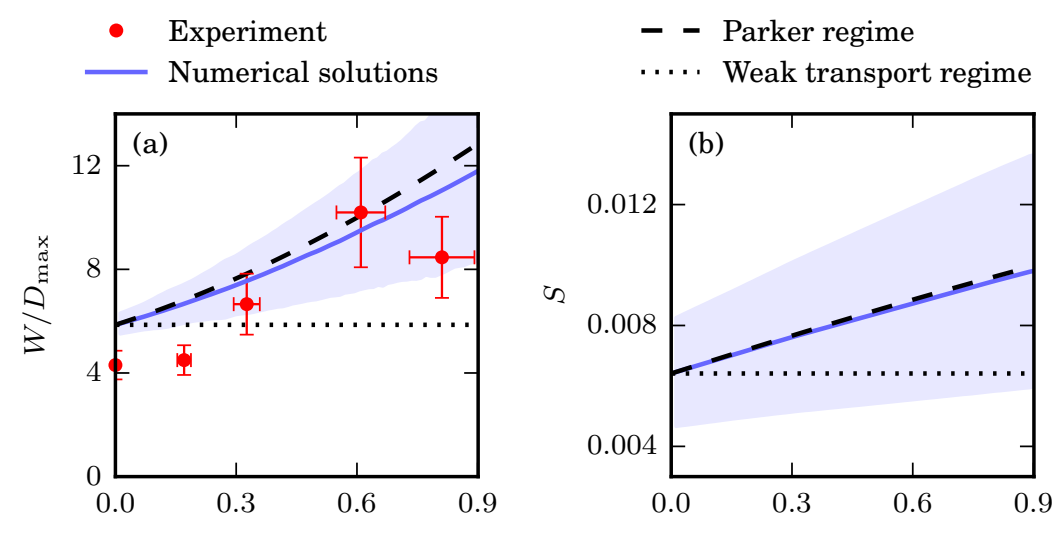

Fig. 3. River properties as a function of the sediment discharge, $Q_{s}$, normalized by the characteristic discharge, $Q_{s}^{*} \approx$ 74 grains s $^{-1}$, given by Eq. 23. Red dots represent the experiments (error bars estimated in SI section S1). Blue lines represent the numerical solutions to Eq. 13 using the experimental parameters (see Table $\mathrm{S} 1$ of the $\mathrm{SI}$ ). Light blue shading corresponds to the uncertainty in the parameter estimates (Table $\mathrm{S} 1$ of the $\mathrm{SI}$ ). The numerical solutions transition from the weak transport regime (black dotted line) to the Parker regime (black dashed line) when $Q_{s} \sim$ $Q_{s, \mathrm{t}} \approx 8.6$ grains s $^{-1}$ (Eq. 29). (a) River
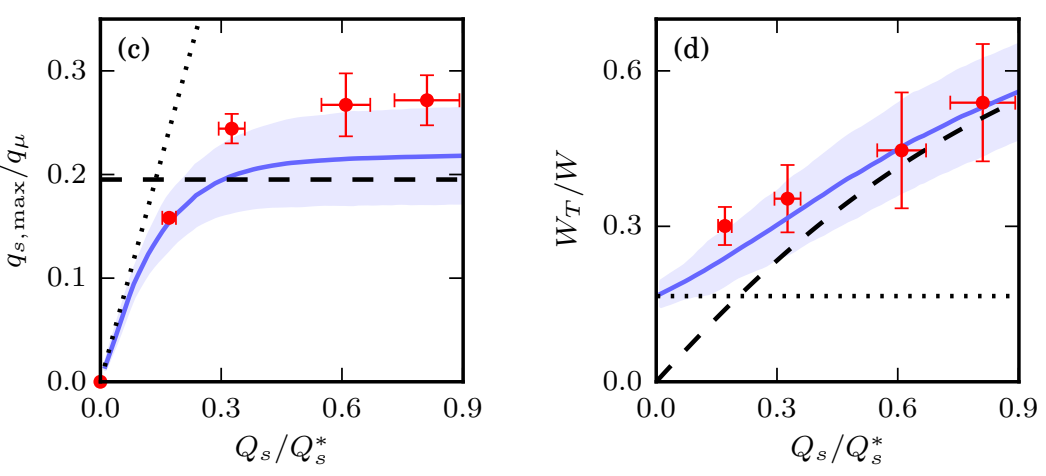
aspect aspect ratio, $W / D_{\max }$. The weak transport regime assumes a fixed bed shape so the aspect ratio is constant. (b) Downstream slope, $S$. The slope is too small for direct measurement. As in panel (a), the fixed bed shape in the weak transport regime leads to a constant slope, while the Parker regime follows from Eq. 24. (c) Normalized maximum sediment flux, $q_{s, \max } / q_{\mu}$, where $q_{\mu}$ is the prefactor of the sediment transport law (Eq. 7). The weak transport regime corresponds to Eq. 27 while the Parker regime corresponds to Eq. 19. (d) Transport width, $W_{T} \equiv Q_{s} /\left\langle q_{s}\right\rangle$, normalized by the total width, $W$. The weak transport regime corresponds to Eq. 28, while the Parker regime follows from Eqs. 19, 20, and 24.

\section{The mechanisms that shape a river}

Keeping in mind the rivers of Abramian et al. (19), the goal of the present paper is to understand how an active laminar river adapts its own depth and sediment flux profiles, $D(y)$ and $q_{s}(y)$, to the fluid and sediment discharges, $Q_{w}$ and $Q_{s}$, it carries. In this section, we will start by reviewing the equations which govern the flow and the transport of sediment in such a river. Throughout the paper, $x$ will represent the downstream, $y$ the cross-stream, and $z$ the vertical coordinate, measured with respect to the surface of the river (Fig. 2a). We restrict our attention to a straight river that is uniform in the $x$-direction. Accordingly, we only need to consider its cross-section in the $(y, z)$ plane.

Stokes flow. In a straight river, the flow is forced by gravity that pushes the fluid down a slope, $S$. This slope is usually very small (for the experiments of Abramian et al. (19), $S \sim 0.01$ ). The laminar flow in such a river obeys the Stokes equation

$$
\nu \Delta u=-g S,
$$

where $u$ is the downstream component of the velocity, $g=$ $9.81 \mathrm{~m} \mathrm{~s}^{-2}$ is the gravitational acceleration, $S$ is the slope in the downstream $(x)$ direction, and $\Delta \equiv \frac{\partial^{2}}{\partial y^{2}}+\frac{\partial^{2}}{\partial z^{2}}$ is the Laplacian operator in the $(y, z)$ plane. The boundary conditions are that the velocity vanishes on the bed $(u=0$ when $z=-D)$ and that there is no shear stress on the free surface $(\partial u / \partial z=0$ when $z=0)$.

The term $g S$ in Eq. 2 is the force driving the fluid flow. In the experiments, the slope is not prescribed a priori. Instead, the river selects it while forming its own bed. It depends on the river's discharges and we cannot prescribe it arbitrarily. 230 Importantly, the Stokes flow is scale-invariant - the flow in two channels of a different size but the same shape looks the same, and one can find one from the other by simple rescaling of lengths and velocity.

If we can find the velocity in the channel by using Eq. 2, we can also get the stress, $\tau$, shearing the bed surface. This stress is proportional to the gradient of $u$ in the direction normal to the bed surface, with the dynamic viscosity, $\rho_{f} \nu$, acting as a constant of proportionality. To get an idea of how the stress depends on the channel shape, we integrate Stokes law, Eq. 2, along the vertical direction, and find an equation for $\tau$ :

$$
\begin{aligned}
\tau & =\left(\rho_{f} g S D+\rho_{f} \nu(\bar{u} D)^{\prime \prime}\right) \cos \phi, \\
\bar{u} & \equiv \frac{1}{D} \int_{-D}^{0} u \mathrm{~d} z
\end{aligned}
$$

where primes denote $y$-derivatives, $\bar{u}$ is the vertically averaged flow velocity, and $\phi$ is the angle between the vector normal to the bed's surface and the vertical (see SI section S2.1 for a detailed derivation). Equation 3 follows without approximation from the Stokes equation. The first term of Eq. 3, $\rho_{f} g S D$, is simply proportional to the weight of the water column. It corresponds to the stress that the fluid would exert on a perfectly flat surface. It ignores the transfer of momentum across stream and we will call it the "shallow-water component", in reference to the celebrated shallow-water approximation. The second term, $\rho_{f} \nu(\bar{u} D)^{\prime \prime}$, accounts for the viscous transfer of momentum across the stream (along $y$ ), and we will call it the "momentum diffusion component". Finally, the term $\cos \phi$ accounts for the orientation of the bed surface. Equation 3 
is not closed - in order to find $\tau$, we still need to solve the Stokes equation for $u$ to get the vertically averaged velocity, $\bar{u}$. Since we hope to bypass the solution of the Stokes equation, Eq. 3 is not very useful in its present form; we will, however, close it by assuming the river is much wider than it is deep (section 3).

Sediment transport. If the forces acting to dislodge sediment grains are too weak, the grains remain trapped on the river bed, and there is no sediment transport (24). The existence of this threshold force is an instance of Coulomb's law of friction (17).

On a flat bed, the fluid acts tangentially to the bed surface, dislodging the grains, while gravity acts normally, anchoring the grains to the bed. In such a case, the sediment flux depends on the so-called Shields parameter, $\theta$, which is proportional to the ratio $F_{f} / F_{g}$ of the fluid force acting on a single grain, $F_{f} \propto \tau d_{s}^{2}$, and the grain's weight, $F_{g} \propto\left(\rho_{s}-\rho_{f}\right) g d_{s}^{3}(24)$ :

$$
\theta \equiv \frac{\tau}{\left(\rho_{s}-\rho_{f}\right) g d_{s}} .
$$

The onset of sediment transport is a complicated phenomenon under active investigation (25-27). However, a simple representation of sediment transport is to assume that on a flat bed, there exists a threshold Shields parameter, $\theta_{t}$, below which there is no sediment transport, while for small deviations above this threshold, the sediment flux, $q_{s}$, increases linearly with the distance to the threshold (28),

$$
q_{s}=q_{0}\left(\theta-\theta_{t}\right) \text { for } \theta>\theta_{t} .
$$

The values of $\theta_{t}$ and $q_{0}$ can be directly measured in experiments. The pre-factor $q_{0}$ is of the order of the ratio of the velocity, $v_{s}$, of a moving grain to its area, $d_{s}^{2}-q_{0} \propto v_{s} / d_{s}^{2}$, where $v_{s}$ is proportional to the Stokes settling velocity, $v_{s} \propto\left(\rho_{s}-\right.$ $\left.\rho_{f}\right) d_{s}^{2} g / \rho_{f} \nu(29,30)$.

On a rounded bed (as in Figs. 2a and b), we cannot simply use the Shields parameter as a criterion for grain motion, since gravity has both a normal and a tangential component with respect to the bed surface. Grains in such a configuration begin to move when the ratio, $\mu$, of tangential forces acting to dislodge the grains to normal forces acting to keep them in place becomes greater than a certain value, $\mu_{t}$, which we can roughly interpret as the friction coefficient (17). We can estimate this friction coefficient independently from $\theta_{t}$ in experiments, e.g. by building a heap of sediment and finding the angle at which its grains begin to topple. Abramian et al. (18) hypothesized that the transport law for the flat bed can be generalized to a curved bed - i.e. that the flux, $q_{s}$, is proportional to the distance of $\mu$ to threshold, $\mu_{t}$ :

$$
q_{s}=q_{\mu}\left(\mu-\mu_{t}\right) \quad \text { for } \quad \mu>\mu_{t} \text {. }
$$

To keep this expression consistent with Eq. 6 for the flat bed, we must have $q_{\mu} \equiv q_{0} \theta_{t} / \mu_{t}$, since, on a flat bed, $\mu=\mu_{t} \theta / \theta_{t}$ (17). Although Eq. 7 is difficult to test independently in an experiment, we will show that it is consistent with the experiments of Abramian et al. (19). Parameters $\theta_{t}, \mu_{t}$, and $q_{0}$ depend on the grain shape and on the Reynolds number at the grain scale. Abramian et al. (18) found them to be $\theta_{t}=$ $0.167 \pm 0.003, \mu_{t}=0.9 \pm 0.2$, and $q_{0}=544 \pm 48$ grains $\mathrm{cm}^{-1} \mathrm{~s}^{-1}$ in their experiments.
To find $\mu$, we need to consider the forces acting on a grain of sediment - the fluid force, $F_{f}$, acts tangentially, while gravity has both a tangential (downhill) component, $F_{g} \sin \phi$, and a component normal to the bed, $F_{g} \cos \phi$. Because the downstream slope of a channel, $S$, is small, the gravitational force is approximately perpendicular to the fluid shear force, $F_{f}$, and the force ratio, $\mu$, is therefore

$$
\mu=\sqrt{\left(\frac{F_{f}}{F_{g} \cos \phi}\right)^{2}+(\tan \phi)^{2}},
$$

The ratio of fluid force to gravity, $F_{f} / F_{g}$, is proportional to the Shields parameter. In particular, we must have $F_{f} / F_{g}=$ $\mu_{t} \theta / \theta_{t}$, since, on a flat bed, $\mu=\mu_{t}$ when $\theta=\theta_{t}$. With this relation, using Eq. 3 for stress, and relating $\phi$ to depth as $\tan \phi=D^{\prime}$, we can express the force ratio $\mu$ from Eq. 8 as

$$
\mu=\sqrt{\left(\frac{\mu_{t} \rho_{f} S}{\theta_{t}\left(\rho_{s}-\rho_{f}\right) d_{s}}\right)^{2}\left(D+\frac{\nu}{g S}(\bar{u} D)^{\prime \prime}\right)^{2}+D^{\prime 2}},
$$

Neglecting the cross-stream momentum diffusion, $(\bar{u} D)^{\prime \prime}$, yields a purely shallow-water model, which Seizilles et al. (17) used to find the shape of inert rivers.

Sediment diffusion. Due to random interactions with the river bed, grains traveling downstream also diffuse laterally, towards areas of the bed where sediment transport is less intense (21). This cross-stream diffusion of sediment opposes gravity, which pulls the grains down towards the center of the channel. Abramian et al. (22) showed that, in equilibrium, the downhill flux of sediment due to gravity is balanced by this uphill diffusive flux of sediment. Like the Boltzmann equilibrium of a gas in a gravitational field, this balance leads to the exponential distribution of the moving grains as a function of the flow depth:

$$
q_{s}=q_{\mathrm{B}} e^{D / \lambda}
$$

The last parameter in this equation, $\lambda$, is the characteristic scale for sediment diffusion, and is analogous to the temperature in a gas. Since sediment diffusion is driven by the bed roughness, $\lambda$ scales with the grain size $\left(\lambda \approx 0.12 d_{s} \pm 20 \%\right.$ (22)).

The prefactor, $q_{\mathrm{B}}$, is the sediment flux at the banks of the river $(D=0)$. Since the flux at the banks is very small compared with the flux at the bottom, $q_{\mathrm{B}}$ does not yield the correct scale for the sediment flux $\left(q_{s, \max } / q_{\mathrm{B}} \sim 10^{23}\right.$ for the experiment with $Q_{s}=60$ grains s$\left.^{-1}\right)$. For this reason, we rewrite Eq. 10 in a more convenient form by defining a parameter $\xi$ with units of depth, such that $q_{\mathrm{B}} \equiv q_{\mu} e^{-\xi / \lambda}$, where $q_{\mu}$ is the prefactor of the sediment transport law, Eq. 7 . In this way, $q_{\mu}$ gives the correct scale for the sediment flux, while $\xi$ is of the order of the maximum depth of an active river $\left(q_{s, \max } / q_{\mu} \sim 0.2\right.$ and $\xi / D_{\max } \sim 1.05$ for the experiment with $Q_{s}=60$ grains $\mathrm{s}^{-1}$ ). As we will see below, the maximum river depth, $D_{\max }$, is generally less than $\xi$, so the maximum flux in a river is typically less than $q_{\mu}$. With this, Eq. 10 becomes

$$
q_{s}=q_{\mu} e^{(D-\xi) / \lambda} .
$$

The parameter $\xi$ controls the intensity of sediment flux and ensures that the sediment discharge is the integral of the flux, $Q_{s}=q_{\mu} \int \exp ((D-\xi) / \lambda) \mathrm{d} y$. As an integration constant, its 
value depends on the discharges transported by the river, but it is not immediately obvious how. A vanishing sediment discharge in rivers corresponds to $\xi \rightarrow \infty$, while finite values of the sediment discharge correspond to smaller values of $\xi$. Sediment transport in a river is significant when the difference, $D_{\max }-\xi$, between the river depth and $\xi$, is of the order of $\lambda$ This is why $\xi$ of active rivers is of the order of the maximum depth, while it is much greater than the depth of inert ones. In the experiments of Abramian et al. (19), $\xi$ is not set a priori, but only becomes measurable after the river has formed, and, in that sense, plays a similar role as the slope, $S$.

Equation 11 relates the sediment flux, $q_{s}$, to the river shape, $D(y)$, and has been confirmed repeatedly in experiments $(21,22)$. We note that, unlike the gas which simply adjusts to the external field, the river selects its own potential (i.e. its own shape), $D(y)$.

\section{Boundary value problem}

The relations for the flow, sediment flux, and sediment diffusion we introduced above combine to determine the equilibrium shape of a river. In particular, the Stokes law, Eq. 2, relates the river depth profile, $D(y)$, to the vertically averaged fluid velocity profile, $\bar{u}(y)$. Then, the sediment flux equations, Eqs. 7 and 9, relate this fluid velocity to the sediment flux profile. Finally, the Boltzmann distribution, Eq. 11, relates the sediment flux back to the depth profile, thereby closing the system of equations for $D(y)$. However, solving these equations simultaneously to get a self-consistent depth and sediment flux profiles is a difficult task - one needs to solve a two-dimensional, partial differential equation with a moving boundary. Instead, in order to make sense of these equations, we propose to approximate the average velocity, $\bar{u}$.

Seizilles et al. (17) showed that the shallow-water approximation accounts for the equilibrium shape of inert laminar rivers. This approximation, which assumes that there is no transfer of momentum between adjacent fluid columns, i.e. that we can neglect the $y$-derivatives of $u$ in the Stokes equation, is exact when the bed is completely flat. It also works well when depth variations occur on length scales that are much longer than the depth itself. In the case of our river, this would apply when the aspect ratio of the river is large. Neglecting the $y$-derivatives in Eq. 2, we find that the vertically averaged shallow-water velocity, $\bar{u}_{\mathrm{sw}}$, is proportional to the square of the depth, a result known as the lubrication approximation (31)

$$
\bar{u}_{\mathrm{sw}}=\frac{g S D^{2}}{3 \nu} .
$$

On a flat bed, where Eq. 12 is exact, the fluid stress, $\tau$, would only contain the shallow-water contribution proportional to depth, $\tau_{\mathrm{sw}}=\rho_{f} g S D$ (Eq. 3). Approximating stress in this way would allow us to close the system of equations for the river shape, in a way similar to Seizilles et al. (17). However, it turns out that keeping only the shallow-water contribution to the stress yields unrealistic profiles for active rivers (i.e. when $Q_{s}>0$ ) (SI section S7.1). Parker (1) first suggested that the cross-stream diffusion of momentum plays an important role for bedload sediment transport in rivers. In line with his suggestion, we keep the momentum diffusion term, $(\bar{u} D)^{\prime \prime}$, in the expression for the stress, but approximate $\bar{u}$ with the shallow-water velocity, $\bar{u}_{\text {sw }}$, given by Eq. 12 . Then, combining
Eqs. 7, 9, and 11, we get an ordinary differential equation expressed solely in terms of the depth and its derivatives:

$$
\sqrt{\frac{S^{2}}{L_{s}^{2}}\left(D+\frac{1}{3}\left(D^{3}\right)^{\prime \prime}\right)^{2}+D^{\prime 2}}-\mu_{t}=e^{(D-\xi) / \lambda},
$$

where we have introduced a length scale of the order of the grain size, $L_{s}$, that is a combination of parameters directly measurable in our experimental setup:

$$
L_{s} \equiv \frac{\theta_{t}\left(\rho_{s}-\rho_{f}\right) d_{s}}{\mu_{t} \rho_{f}} .
$$

We discuss this approximation in detail in the SI sections S2.2 and $\mathrm{S} 2.3$, where we show that it is the first term in a series expansion for large aspect ratio, $W / D_{\max }$ - it corrects the shallow water stress with a term of order $D_{\max }^{2} / W^{2}$. There, we also show that the contribution of momentum diffusion, $\left(D^{3}\right)^{\prime \prime} / 3$, in Eq. 13 is of the same order as the contribution of gravity, $D^{\prime}$, so that it should not be neglected in a selfconsistent model of a river (SI section S2.2). Recognizing that momentum diffusion is essential to form active rivers, and finding a suitable approximation for it, is a major theoretical contribution of our paper. In principle, Eq. 13 could fail to be a meaningful approximation of the stress in a channel with an aspect ratio of order one, but, in our case, it meaningfully corrects the stress for rivers under all experimental conditions we tested (even in the case of inert rivers with $W / D_{\max } \sim 4$ ).

Equation 13 is an ordinary differential equation. To solve this second-order problem, we need to specify two boundary conditions. For a solution of Eq. 13 to be a river, the depth needs to vanish on the banks and the center needs to be flat. Therefore, Eq. 13 is a boundary value problem (BVP) with boundary conditions $D(y=-W / 2)=0$ and $D^{\prime}(y=0)=0$. There are several parameters that enter our equation, some of which are directly measurable in our experimental setup $\left(\mu_{t}\right.$, $\lambda$, and $L_{s}$ ), while others depend implicitly on the discharges of fluid and sediment and become apparent only after the river has formed ( $S$ and $\xi$ ). Although the river width, $W$, is unknown a priori, it is not an independent parameter - it can be inferred through solving Eq. 13 for a given choice of other parameters (SI section S3.1). We emphasize that Eq. 13 describes the equilibrium river profile, and, therefore, does not convey anything about transient, time-dependent processes that occur as the river approaches the equilibrium.

\section{Dependence on water and sediment discharge}

If we choose the parameters $\mu_{t}, \lambda, L_{s}, S$ and $\xi$, we can numerically solve Eq. 13 to get a unique river profile, $D(y)$ (SI section S3.1). However, since $S$ and $\xi$ are not directly measurable in our experiment, we cannot immediately determine the shape of the river by simply prescribing the discharge of fluid and sediment in the same way as we would in an experiment. The dependence of $S$ and $\xi$ on the discharges is complicated, and, on the theoretical grounds, we can only say that the inert river, $Q_{s}=0$, corresponds to $\xi \rightarrow \infty$, while active rivers correspond to smaller values of $\xi$. Nevertheless, we can find this dependence numerically as follows. For each solution, $D(y)$, of our equation that corresponds to a particular choice of $S$ and $\xi$, we can find the discharges of fluid and sediment as

$$
Q_{w}=\int_{-W / 2}^{W / 2} \frac{g S D^{3}}{3 \nu} \mathrm{d} y \quad, \quad Q_{s}=\int_{-W / 2}^{W / 2} q_{\mu} e^{(D-\xi) / \lambda} \mathrm{d} y,
$$


where we relate the fluid discharge, $Q_{w}=\int D \bar{u} \mathrm{~d} y$, to the depth profile by approximating $\bar{u}$ with the shallow-water velocity, $\bar{u}_{\mathrm{sw}}$ (Eq. 12), and use the Boltzmann distribution (Eq. 11) to relate the sediment flux to depth. Keeping $\mu_{t}$, $\lambda$, and $L_{s}$ fixed to their experimental values, $Q_{w}$ and $Q_{s}$ are only functions of the parameters $S$ and $\xi$. Inverting these relations numerically yields the model parameters as functions of the discharges of water and sediment, $S\left(Q_{w}, Q_{s}\right)$ and $\xi\left(Q_{w}, Q_{s}\right)$. This allows us to directly compare our theory to the experiments (SI section S3.2). We find that the theoretical cross-sections and sediment flux profiles resemble their experimental counterparts, without any fitting parameter (Fig. 2).

Encouraged by this result, we now describe how our theoretical rivers depend on $Q_{w}$ and $Q_{s}$ (see also SI section S3.3). As we increase the water discharge, $Q_{w}$, the width and depth of the river increase approximately as $Q_{w}^{1 / 3}$, while its slope decreases roughly as $Q_{w}^{-1 / 3}$ (SI Fig. S5f), in accordance with the result of Seizilles et al. (17) for inert rivers. This $1 / 3$ exponent is a signature of the laminar flow in our rivers, in contrast with natural turbulent ones which scale with the $1 / 2$ exponent of the empirical Lacey's law $(10,11)$. Though the size of a river in our model may vary by orders of magnitude under varying $Q_{w}$, its shape, described for example by the aspect ratio, does not change much unless the river transports a significant amount of sediment. On the other hand, increasing $Q_{s}$ while keeping $Q_{w}$ fixed makes the river wider and shallower, while affecting its overall scale only slightly. In short, the water discharge sets the size of the river, while the sediment discharge sets its shape.

In Fig. 3, we show that our predictions fall within the uncertainty range of observations of Abramian et al. (19). The aspect ratio and the transport width, $W_{T}$, increase with sediment discharge in both the model and the experiments (Figs. 3a and d). The sediment flux increases and saturates for large sediment discharge (Fig. 3c). This explains why the river becomes wider as we increase $Q_{s}$ - if the sediment flux, $q_{s}$, saturates, the river needs to widen to accommodate a larger sediment discharge. At the same time, this widening forces the river to become shallower in order to maintain a constant fluid discharge, $Q_{w}$, so that its overall size does not change much while its aspect ratio grows. The simple, nearly linear relationship between the aspect ratio and the sediment discharge shown in Fig. 3a means that this basic geometric property of the river shape can be used to infer the sediment load, at least in the case of straight, laminar, single thread rivers.

Since the downstream slope, $S$, is very small, it cannot be measured directly in the experiments. Nevertheless, our theory makes a prediction for it, which we show in Fig. 3b: the predicted slope is of the order of 0.01 , and increases almost linearly with $Q_{s}$.

\section{Inert, active, and limiting river}

We can simplify our model by making Eq. 13 non-dimensional, thereby reducing the number of parameters that represent the river. To that end, we rescale all lengths by $L_{s} / S$

$$
\tilde{y} \equiv \frac{y S}{L_{s}} \quad, \quad \tilde{D} \equiv \frac{D S}{L_{s}} \quad, \quad \tilde{\lambda} \equiv \frac{\lambda S}{L_{s}} \quad, \quad \tilde{\xi} \equiv \frac{\xi S}{L_{s}} .
$$
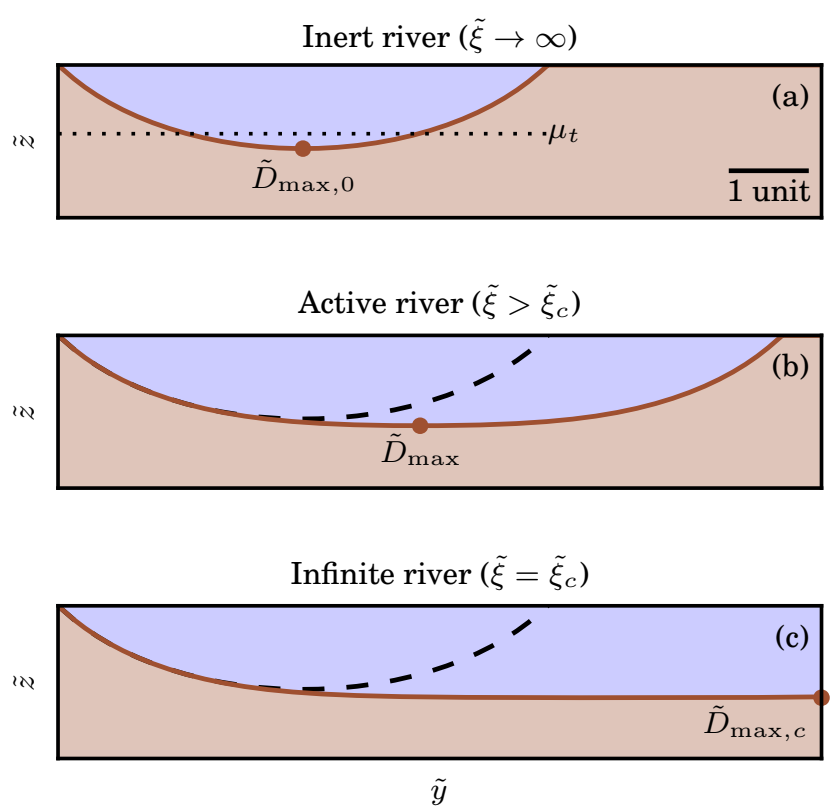

Fig. 4. River solutions in the non-dimensional $\tilde{y}$ - $\tilde{z}$ space for $\mu_{t}=0.9, \tilde{\lambda}=0.1$, and varying $\tilde{\xi}$. Black dashed lines in panels (b) and (c) represent the inert river from the upper panel. Aspect ratio is preserved. (a) Inert river $(\tilde{\xi} \rightarrow \infty)$. Brown dot marks the non-dimensional inert river depth, $\tilde{D}_{\max , 0}$. This depth is greater than the friction coefficient, $\tilde{D}_{\max , 0}>\mu_{t}$, marked by the horizontal dotted line. (b) Active river $\left(\tilde{\xi}=1.33>\tilde{\xi}_{c}\right)$. Brown dot marks the depth, $\tilde{D}_{\max }$. (c) Infinite limiting river $\left(\tilde{\xi}=1.3237 \approx \tilde{\xi}_{c}\right)$. Brown dot marks the limiting depth, $\tilde{D}_{\max , c}$.

In terms of these non-dimensional parameters, Eq. 13 becomes:

$$
\sqrt{\left(\tilde{D}+\frac{1}{3}\left(\tilde{D}^{3}\right)^{\prime \prime}\right)^{2}+\tilde{D}^{\prime 2}}-\mu_{t}=e^{(\tilde{D}-\tilde{\xi}) / \tilde{\lambda}}
$$

where, now, the primes stand for derivatives with respect to $\tilde{y}$. The non-dimensional depth, $\tilde{D}$, is of order one, regardless of the size of the original river. Therefore, Eq. 17 describes the river shape, while the ratio $L_{s} / S$ sets its size. The river shape depends on only three non-dimensional parameters $\mu_{t}, \tilde{\lambda}$, and $\tilde{\xi}$.

When $\tilde{\xi} \rightarrow \infty$ (Fig. 4a, SI section S4.3), the river becomes inert as the exponential on the right-hand side of Eq. 17 vanishes. In this case, the dependence on $\tilde{\xi}$ and $\tilde{\lambda}$ vanishes, so the river shape depends only on the friction coefficient, $\mu_{t}$. Since the friction coefficient is a fixed property of the sediment grains, the fluid discharge, $Q_{w}$, cannot change the shape of such a river. Instead, the fluid discharge can only affect its size by changing the scale factor, $L_{s} / S$. Physically, this is because the laminar flow is scale-free, which makes the inert river shape independent of its size. An inert river, thus, reaches a maximum depth $\tilde{D}_{\max , 0}\left(\mu_{t}\right)$. To calculate its value, we need to numerically solve Eq. 17, but unlike the complete theory of section 3 , this is a straightforward problem since it depends on a single, directly measurable parameter. For $\mu_{t}=0.9$ which corresponds to the experiments, we numerically find $\tilde{D}_{\max , 0}\left(\mu_{t}\right) \approx 1.1$. From Eq. 17 , we can express this depth as $\tilde{D}_{\max , 0}=\mu_{t}-\left.\frac{1}{3}\left(\tilde{D}^{3}\right)^{\prime \prime}\right|_{\text {center }}$, since the gravity contribution, $\tilde{D}^{\prime}$, vanishes at the river center. Thus, the momentum diffusion contribution to the stress (the term $\frac{1}{3}\left(\tilde{D}^{3}\right)^{\prime \prime}$ in Eq. 17) ensures that the dimensionless inert river depth is greater than the friction coefficient $\left(\tilde{D}_{\max , 0}>\mu_{t}\right)$ - had we ignored the momentum diffusion, the inert river 
Weak transport regime $\left(Q_{s}=5\right.$ grains $\left.\mathrm{s}^{-1}\right)$
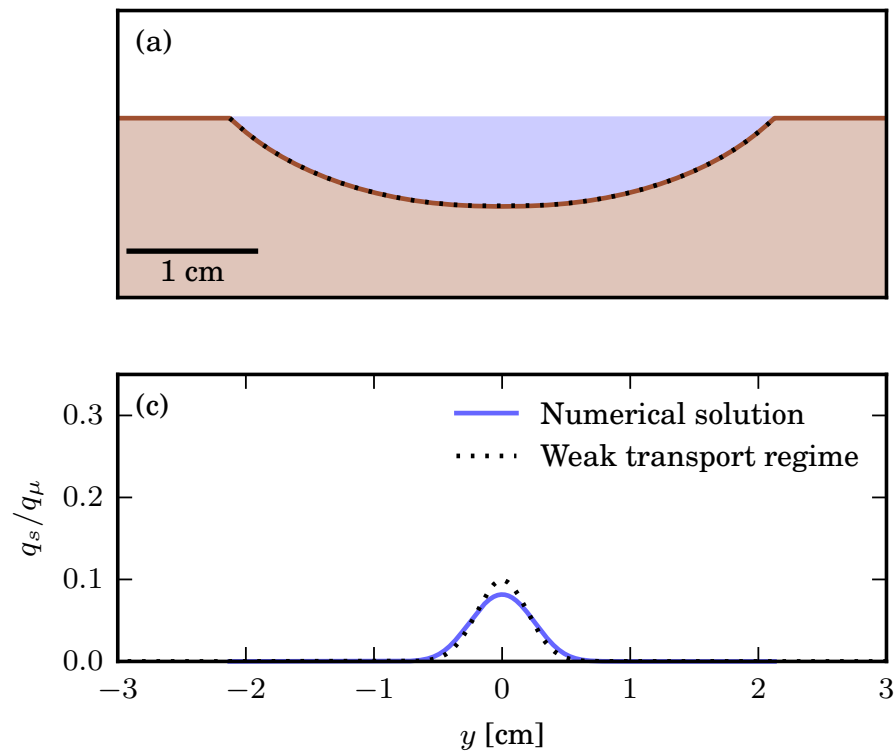

Parker regime $\left(Q_{s}=40\right.$ grains $\left.\mathrm{s}^{-1}\right)$
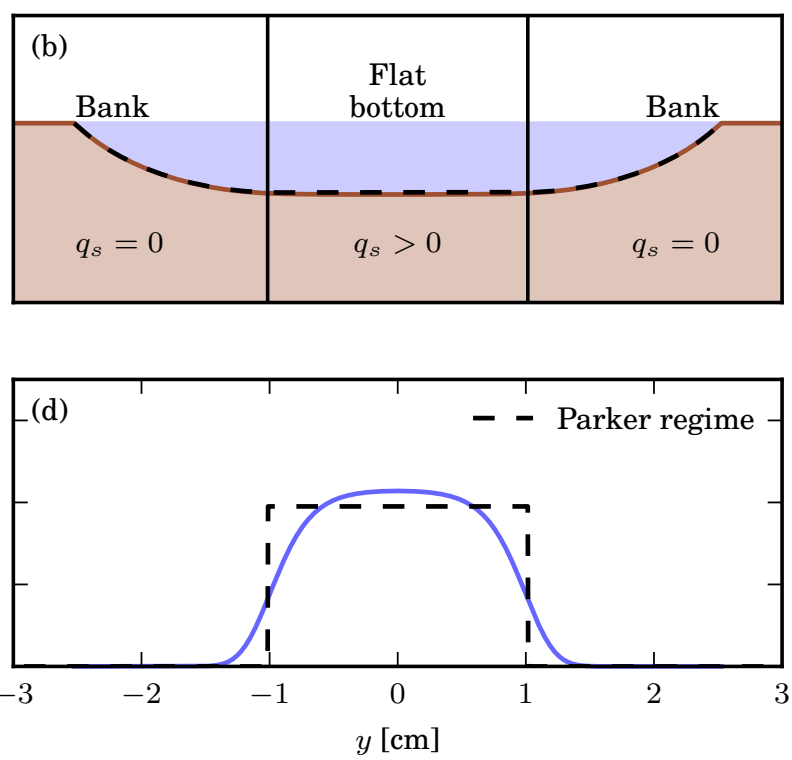

Fig. 5. Asymptotic regimes. Black dashed lines correspond to the Parker regime while the black dotted lines correspond to the weak transport regime. Top row panels ((a) and (b)) show river depth profiles in our model. The brown lines are numerical solutions of Eq. 13 for two values of sediment discharge, $Q_{s}$. The numerical and asymptotic depth profiles are not distinguishable by eye and the error is of the order $\lambda$ (or about $2 \%$ of the maximum depth) - the maximum deviation of the asymptotic approximation from the numerical solution is about $0.05 \mathrm{~mm} \approx 0.5 \lambda$ in panel (a) and about $0.15 \mathrm{~mm} \approx 1.5 \lambda$ in panel (b). The vertical black lines in panel (b) split the river in three parts used to construct the Parker river. Lower row panels ((c) and (d)) show the dimensionless sediment flux profiles, $q_{s} / q_{\mu}$, that correspond to upper panels. Blue lines are numerical solutions.

depth would have been exactly $\mu_{t}$. Physically, the diffusion of momentum relieves some of the stress from the river center, so, to remain at the threshold of sediment motion, the river has to be deeper than it would be without momentum diffusion. In the next section, we will show that this fact is crucial for the transport of sediment.

As $\tilde{\xi}$ decreases to finite values, the river becomes active (Fig. 4b). The banks of such a river largely retain the shape of the inert one, but its bottom part, which carries most of the sediment, widens, and the discharge of sediment increases. For a particular value of $\tilde{\xi}$, say $\tilde{\xi}_{c}$, which depends on $\mu_{t}$ and $\tilde{\lambda}$, the river becomes infinitely wide and transports an infinite amount of sediment (Fig. 4c, SI section S4.2). Such a river has a finite, well-defined depth, $\tilde{D}_{\max , c}\left(\mu_{t}, \tilde{\lambda}\right)$. This means that, for given values of $\tilde{\lambda}$ and $\mu_{t}$, there exists a river-solution with a highest possible sediment flux, $q_{s, c}=q_{\mu}\left(\tilde{D}_{\max , c}-\mu_{t}\right)$. The existence of this limiting flux explains the saturation of $q_{s}$ for large values of total sediment discharge, $Q_{s}$, that we see in Fig. 3c. It also means that, in our model, the distance to threshold in a river, $\mu-\mu_{t}$, is always less than $\tilde{D}_{\max , c}-\mu_{t}$. Numerically, we find $\tilde{D}_{\max , c}-\mu_{t} \approx 0.22$ for experimental parameters $\left(\mu_{t}=0.9\right.$ and $\left.\tilde{\lambda}=0.02\right)$. In the next section, we will estimate the limiting flux, $q_{s, c}$, by assuming sediment diffusion is weak $(\lambda \rightarrow 0)$, in which case $q_{s, c}$ only depends on the friction coefficient, $\mu_{t}$.

\section{The Parker regime}

Moving grains accumulate at the bottom of the river due to gravity, while they climb back onto the banks by diffusion (22). The Boltzmann distribution, Eq. 11, implies that diffusion can pull the grains up by a height that is of the order of the length scale $\lambda$. Therefore, the region of the bed over which transport occurs has a depth that is within several $\lambda$ of the maximum, $D_{\max }$. Since $\lambda$ is small (less than the grain size), rivers that transport a significant amount of sediment need a wide, and essentially flat bottom. Moreover, a small $\lambda$ means the sediment transport decreases rapidly towards the banks, so the banks are nearly inert and, thus, close to the threshold of sediment motion. If, following this reasoning, we neglect sediment diffusion altogether by taking the limit $\lambda \rightarrow 0$, the river sharply separates into a flat, active bottom and curved, inert banks (Fig. 5b). We will call this simplified configuration the "Parker regime," after Parker (1) who constructed a similar model for natural gravel-bed rivers. The limit $\lambda \rightarrow 0$ is equivalent to assuming that the fluid discharge is large (to ensure that $\lambda$ is small compared with the width of the inert banks), and that the sediment discharge is large (to ensure that $\lambda$ is small compared with the width of the active, flat bottom).

We begin the investigation of this regime by first finding the depth, $D_{\max }^{(P)}$, of a Parker river (denoted by the superscript $(P))$. The banks in this approximation are inert and, thus, satisfy our model, Eq. 17, with the right-hand side set to zero. Therefore, their non-dimensional depth matches that of an inert river, $\tilde{D}_{\max , 0}\left(\mu_{t}\right)$, and we can set, $\tilde{D}_{\max }^{(P)}=\tilde{D}_{\max , 0}\left(\mu_{t}\right)$. In dimensional units, this becomes

$$
D_{\max }^{(P)}=\frac{L_{s} \tilde{D}_{\max , 0}\left(\mu_{t}\right)}{S^{(P)}} .
$$

We note that this dimensional depth of a Parker river differs from that of an inert river, since the slope of a Parker river, $S^{(P)}$, is different from the slope of an inert river, $S_{0}$ - these slopes depend on the shape of the entire channel, not only on the banks. The non-dimensional inert river depth, $\tilde{D}_{\max , 0}$, depends only on the friction coefficient, $\mu_{t}$ (section 5). For 
this reason, the river depth given by Eq. 18 is inversely proportional to its slope, $D_{\max }^{(P)} \propto 1 / S^{(P)}$, regardless of the fluid and sediment discharges. This is consistent with the original model of Parker, as well as with observations in natural rivers $(1,32)$.

Once we know the depth of a Parker river, we can find its sediment flux. Since the bottom is flat, the cross-sectional profile of the sediment flux is a rectangle of height $q_{s}^{(P)}$ and width $W_{T}^{(P)}$ (black dashed line in Fig. 5d). The flat bottom feels only the shallow-water component of the stress so the force ratio on the bottom is $\mu=\tilde{D}_{\max }^{(P)}$ (Eq. 9). According to the transport law, Eq. 7, this yields a sediment flux

$$
\frac{q_{s}^{(P)}}{q_{\mu}}=\tilde{D}_{\max , 0}\left(\mu_{t}\right)-\mu_{t},
$$

where we used $\tilde{D}_{\max }^{(P)}=\tilde{D}_{\max , 0}\left(\mu_{t}\right)$. Therefore, the sediment flux, and, correspondingly, the distance to threshold, $\mu-\mu_{t}$, depend only on the friction coefficient, $\mu_{t}$, and have the same value regardless of the discharges of fluid and sediment - this is the gist of the Parker regime. Numerically solving Eq. 17 for an inert river (with $\mu_{t}=0.9$ ), we find $\tilde{D}_{\max , 0}-\mu_{t} \approx 0.2$. The sediment flux of a Parker river, $q_{s}^{(P)}$, is an approximation of the limiting flux, $q_{s, c}$, we discussed in section 5 - in fact, $q_{s}^{(P)}$ is the limit of $q_{s, c}$ as $\lambda \rightarrow 0$. For typical experimental parameters $\left(\mu_{t}=0.9\right.$ and $\left.\tilde{\lambda}=0.02\right), q_{s, c}$ is less than $10 \%$ higher than $q_{s}^{(P)}$, so the Parker regime approximates the numerical solution well.

The sediment flux $q_{s}^{(P)} \approx 0.2 q_{\mu}$ we find for a laminar river corresponds to a fluid-induced stress on the river bottom that is about $22 \%$ higher than critical. Interestingly, this value of the stress is comparable to observations in natural rivers, and to the original Parker's theory for turbulent rivers $(1,33)$. There is no reason to expect that this proportion should be exactly the same for laminar and turbulent flows. However, that it is independent from the water and sediment discharges, and of order one, is likely not a coincidence. The scale-independence of the flow ensures that the bank shape (i.e. $\left.\tilde{D}_{\max , 0}\left(\mu_{t}\right)\right)$ is independent of the discharges (section 5 ). Thus, the discharge-independent sediment flux likely results from the scale-independence of the flow, under both laminar and turbulent conditions.

The difference $\tilde{D}_{\max , 0}-\mu_{t}$ vanishes in the classical shallowwater approximation (section 5). As a consequence, if we ignored momentum diffusion, the river could not carry any sediment - sediment transport in the Parker regime is only possible because momentum diffuses across the stream. Indeed, this tends to homogenize the distribution of shear stress over the bed, especially on the banks, where the bed is curved. As a consequence, the deeper parts of the banks hand over some of the momentum to the shallower parts of the banks. This means that, in order to stay at the threshold of sediment motion, the banks need to be deeper than they would be in the absence of momentum diffusion. This increased depth then causes excess stress on the flat river bottom, which only feels the shallow-water component of the stress, thereby driving sediment transport (SI section S7). This is why we need to keep track of momentum diffusion, even in a minimal model of a river.

The weakness of sediment diffusion, characterized by a small diffusion length, $\lambda$, ensures that rivers remain close to the threshold. For a small but finite $\lambda$, we numerically find that the maximal distance to threshold is approximately
$\tilde{D}_{\max , c}-\mu_{t} \approx \tilde{D}_{\max , 0}\left(\mu_{t}\right)-\mu_{t}+S \lambda / L_{s}$ (SI section S4.4). The term $S \lambda / L_{s}$ is negligible for large rivers with a small slope, such as the ones in the experiments we are considering $\left(S \lambda / L_{s} \approx 0.022\right.$ for highest experimental $\left.Q_{s}\right)$. The fact that rivers tend towards the Parker regime as the fluid and sediment discharges increase is, thus, the reason they do not exceed the threshold significantly more than $\tilde{D}_{\max , 0}\left(\mu_{t}\right)-\mu_{t}$. In short, it is the combination of significant momentum diffusion with weak bedload diffusion that maintains the laboratory rivers of Abramian et al. (19) near the threshold.

Once we have identified the sediment flux, $q_{s}^{(P)}$, all other properties follow straightforwardly. In particular, we can get the width of the active bottom, $W_{T}^{(P)}$, as

$$
W_{T}^{(P)}=Q_{s} / q_{s}^{(P)} .
$$

The total width of a Parker river, $W^{(P)}=W_{T}^{(P)}+W_{0}^{(P)}$, is then the sum of $W_{T}^{(P)}$ and the bank width, $W_{0}^{(P)}=\tilde{W}_{0} L_{s} / S^{(P)}$. Here, $\tilde{W}_{0}$ is the non-dimensional width of an inert river that is only a function of $\mu_{t}$ (numerically, we find $\tilde{W}_{0} \approx 6.4$ for $\left.\mu_{t}=0.9\right)$.

From here, we can find the aspect ratio of a Parker river as

$$
\frac{W^{(P)}}{D_{\max }^{(P)}}=\frac{\tilde{W}_{0}}{\tilde{D}_{\max , 0}}+\frac{Q_{s}}{q_{s}^{(P)} D_{\max }^{(P)}} .
$$

This equation shows how the geometry of a river can be used to infer its sediment load. Namely, from Eq. 21, the sediment discharge is

$$
Q_{s}=q_{s}^{(P)} D_{\max }^{(P)}\left(\frac{W^{(P)}}{D_{\max }^{(P)}}-\frac{\tilde{W}_{0}}{\tilde{D}_{\max , 0}}\right) .
$$

The quantities $q_{s}^{(P)}$ and $\tilde{W}_{0} / \tilde{D}_{\max , 0}$ are universal in that they only depend on the properties of the sediment and the general properties of the flow (such as its laminarity). As such, they are independent of the discharges of fluid and sediment. All other quantities on the right hand side of Eq. 22 are geometric $\left(D_{\max }^{(P)}\right.$ and $\left.W^{(P)}\right)$. Therefore, one can estimate the sediment load of a river in the Parker regime by simply measuring its width and depth. Equation 22 follows from general considerations that allow the Parker regime to exist - such as, for example, that the river splits into an active bottom and inert banks whose shape is independent of the discharges. It is likely that these conditions also apply to turbulent rivers. So, we speculate that Eq. 22 holds for natural rivers in the Parker regime, although with different values of $q_{s}^{(P)}$ and $\tilde{W}_{0} / \tilde{D}_{\max , 0}$. On an ensemble of rivers with varying fluid and sediment discharge, $q_{s}^{(P)}$ would represent the maximum observed sediment flux, while $\tilde{W}_{0} / \tilde{D}_{\max , 0}$ would be the minimum observed aspect ratio. So, to estimate the sediment load of a natural river, one could begin by estimating the minimum aspect ratio and maximum sediment flux on a large dataset of rivers, and then measuring the width and depth of a particular river. We demonstrate the validity of this method on our experimental dataset in the SI section $\mathrm{S} 6$.

The transport width of a Parker river becomes comparable to the river size when the sediment discharge is $Q_{s} \approx q_{s}^{(P)} L_{s} / S^{(P)}$. This defines a characteristic discharge in the Parker regime, $Q_{s}^{*}$ :

$$
Q_{s}^{*}=q_{\mu}\left(\frac{\nu Q_{w}}{g L_{s}}\right)^{1 / 3},
$$


where we approximated the slope with that of an inert river (Eq. S38 of the SI) and neglected dimensionless factors of order one. When the sediment discharge is much greater than $Q_{s}^{*}$, the aspect ratio of a river grows with the sediment discharge, while for $Q_{s}$ much smaller than $Q_{s}^{*}$, it becomes that of the inert river. In the experiments, we find $Q_{s}^{*} \approx 74$ grains s $^{-1}$. Interestingly, this value is close to the discharge $Q_{s} \approx 90$ grains s $^{-1}$ at which the experimental rivers destabilize into braids, suggesting that $Q_{s}^{*}$ may define an upper bound for the sediment load a single channel can carry. Determining this would, however, require an analysis that is beyond the scope of this paper.

Finally, to find the slope of the Parker river, $S^{(P)}$, we first compute its water discharge, $Q_{w}$ (Eq. 15). The water discharge of a Parker river is a sum of the the bank and the flat bottom contributions. In particular, we find (SI section S5)

$$
Q_{w}=\frac{g L_{s}^{4}}{\nu S^{(P) 3}}\left(\tilde{Q}_{w, 0}+\frac{Q_{s} S^{(P)} \tilde{D}_{\max , 0}^{3}}{3 q_{s}^{(P)} L_{s}}\right)
$$

where $\tilde{Q}_{w, 0} \equiv \frac{1}{3} \int_{-\tilde{W}_{0} / 2}^{\tilde{W}_{0} / 2} \tilde{D}_{0}^{3} \mathrm{~d} \tilde{y}$, is the dimensionless discharge of an inert river with a depth profile $\tilde{D}_{0}(\tilde{y})$, and is, again, only a function of $\mu_{t}\left(\tilde{Q}_{w, 0} \approx 1.22\right.$ for $\left.\mu_{t}=0.9\right)$. The above equation can be inverted to get $S^{(P)}$ as a function of $Q_{w}$, $Q_{s}$, and other measurable parameters, but, since the inverted expression is cumbersome, we do not show it here. To leading order, $S^{(P)} \propto Q_{w}^{-1 / 3}$, so the Parker river inherits the basic scaling of laminar rivers (17).

In Figs. 5b and d, we show that the cross-section and sediment flux profiles of rivers in the Parker regime capture well the numerical solutions of our model, Eq. 13, when the sediment discharge is large. In Fig. 3, we compare the Parker river properties to numerical solutions of the full theory and experiments (black dashed lines in Fig. 3). The slope and shape of numerical solutions are well approximated by the Parker regime for the entire range of sediment discharge (Figs. $3 \mathrm{a}$ and $\mathrm{b})$. Conversely, the sediment flux profile $\left(q_{s}\right.$ and $\left.W_{T}\right)$ for the Parker river is a good approximation of the full theory only when the sediment discharge is large enough. This is not surprising, since, according to the Boltzmann distribution, Eq. 11 , the flux is a sensitive function of the depth so, to get a reasonable estimate of the flux, we need to estimate the depth accurately with a precision that is of the order of the diffusion length, $\lambda$.

When the sediment discharge is small, sediment diffusion becomes important, and the Parker regime cannot account for the sediment transport (Fig. 5c). In the next section, we consider this weak transport regime.

\section{Weak transport regime}

When the sediment discharge is small, the sediment flux concentrates about the center of the river, and does not significantly alter its shape. The sediment flux profile in this case is analogous to the density of an ideal gas in a fixed potential - the fixed bed shape sets the potential well in which the traveling grains distribute themselves.

A random walker that makes steps of length $\lambda$ in a fixed potential well with a characteristic size $L$ would spend the majority of its time moving around in an area with a size of the order of $\sqrt{\lambda L}$. Therefore, we expect the sediment grains in this weak transport regime to concentrate in a region of a size $W_{T} \sim \sqrt{\lambda L_{s} / S_{0}}$, where $L_{s} / S_{0}$ is the characteristic size of an inert river. As a consequence, the sediment flux would be about $q_{s} \sim Q_{s} \sqrt{S_{0} / \lambda L_{s}}$. Thus, unlike the Parker river which changes its width to accommodate its sediment load, the weak-transport river adjusts its sediment flux.

We can formalize this argument by first assuming that the depth profile is approximately that of an inert river, $D_{0}$. Close enough to the center, we can approximate this depth with a parabola,

$$
D_{0} \approx D_{\max , 0}-\frac{\kappa}{2} y^{2},
$$

where $\kappa \equiv-\left.D_{0}^{\prime \prime}\right|_{\text {center }}$ is the curvature of the bed at the center. If only this quadratic part of the depth profile is relevant, the Boltzmann distribution of traveling grains, Eq. 11, becomes a Gaussian:

$$
q_{s}=q_{s, \max } e^{-\frac{\kappa}{2 \lambda} y^{2}} .
$$

Here, $q_{s, \max }$ is a constant that depends on $q_{\mu}, \lambda$, and $\xi$. This approximation is valid when the sediment discharge is small enough to leave the depth profile unaltered, and when the fluid discharge is large enough to keep $\sqrt{\lambda / \kappa}$ small compared with the river size.

To specify the sediment flux profile, we first relate the curvature at the river bottom, $\kappa$, to the depth of an inert river, $D_{\max , 0}$, using Eq. 13. Then, by integrating the sediment flux profile, Eq. 26, we can find the maximum flux, $q_{s, \max }$, and the transport width, $W_{T}=Q_{s} /\left\langle q_{s}\right\rangle$, as functions of $Q_{s}$ (we find $\left\langle q_{s}\right\rangle$ through Eq. 1):

$$
\begin{aligned}
& q_{s, \max }=Q_{s} \sqrt{\frac{S_{0}\left(\tilde{D}_{\max , 0}-\mu_{t}\right)}{2 \pi \lambda L_{s} \tilde{D}_{\max , 0}^{2}}}, \\
& W_{T}=\sqrt{\frac{4 \pi \lambda L_{s} \tilde{D}_{\max , 0}^{2}}{S_{0}\left(\tilde{D}_{\max , 0}-\mu_{t}\right)}},
\end{aligned}
$$

where $S_{0}$ can be estimated from the fluid discharge, Eq. 15, ${ }_{797}$ using the inert river profile (Eq. S38 of the SI). We can see that $q_{s, \max } \propto Q_{s} \sqrt{S_{0} / \lambda L_{s}}$ and $W_{T} \propto \sqrt{\lambda L_{s} / S_{0}}$, as anticipated.

Unlike the Parker regime, the weak transport regime requires sediment diffusion - it does not exist when $\lambda$ vanishes. Figures 3 and 5 show that the sediment flux profile in the numerical model transitions smoothly from the weak transport regime to the Parker regime. This transition happens when $Q_{s}$ approximately equals $q_{\mu} \sqrt{\lambda L_{s} / S_{0}}$, at which point the weak transport sediment flux overcomes the limiting flux of the Parker regime. This defines a transitional sediment discharge, $Q_{s, \mathrm{t}}$, given by

$$
Q_{s, \mathrm{t}}=q_{\mu}\left(\frac{\lambda^{3} \nu Q_{w}}{g L_{s}}\right)^{1 / 6} .
$$

For the experiments we are considering, we find $Q_{s, \mathrm{t}} \approx$

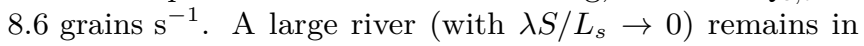
the weak transport regime when $Q_{s} \ll Q_{s, \mathrm{t}}$, and enters the Parker regime if $Q_{s} \gg Q_{s, \mathrm{t}}$. When $\lambda$ vanishes, the transitional discharge tends to zero, and the river is always in the Parker regime. Interestingly, the experiments span both regimes - one experimental run of Abramian et al. (19) has $Q_{s} \approx 12.6$ grains s $^{-1}$, comparable to $Q_{s, \mathrm{t}}$. This means that $\lambda$ in the experiments is small enough for the two regimes to be valid approximations, but still large enough for the weak transport regime to be visible. 


\section{Conclusions}

In this paper, we relied on a recent experimental success in obtaining single-thread laminar rivers, and we developed a physical theory that correctly represents the shape of a river as a function of its water and sediment discharges. In steadystate, the balance between gravity and the stress induced by the fluid flow, along with the diffusion of sediment across the channel, determines the shape of the river. We greatly simplify the problem of calculating the fluid stress, which in general depends on the entire channel shape, by relating it to the local river depth and its derivatives only. Although this model can be numerically solved relatively easily, the relationship between the river properties and the discharges of water and sediment is not immediately obvious. Fortunately, when the sediment discharge is small or large, the relationships between the properties of the river and its discharges reduce to simple algebraic expressions. When the sediment load of a river is large, we find that the diffusion of momentum across the stream generates an excess of stress on the river bottom, which drives sediment transport. Momentum diffusion, thus, plays a key role in determining the shape of the channel, in accordance with the model originally proposed by Parker (1). This is not the case in the weak transport regime, which relies on the diffusion of sediment.

Rivers in our model never deviate much from the threshold of sediment motion. As their sediment discharge increases, the sediment flux approaches a maximum, which forces the river to widen and get shallower. This saturation of the sediment flux results from the weakness of sediment diffusion and the scale-independence of the flow. Most likely, natural rivers also meet these conditions, which is why the original model of Parker (1) has proven to be a fair representation of natural, gravel-bed rivers. According to our theory, however, there exists another, small discharge regime in which a river's shape is independent of its sediment discharge, while its sediment flux is proportional to it. To our knowledge, this regime remains to be identified in the field.

Although our model is aimed at a relatively narrow subset of rivers (straight, laminar rivers that transport uniform, noncohesive sediment as bedload and with constant water and sediment discharge), it is tempting to extrapolate it to natural rivers which have been observed to maintain their channel close to the threshold of sediment motion. The common explanation for this is that hillslope processes, which feed rivers with sediment, are slow, so that rivers carry only a small sediment load. We show that this is not necessarily true our rivers are always close to the threshold, regardless of the sediment discharge, due to the nature of the fluid-sediment interaction.

Increasing the sediment discharge significantly beyond the last experimental point of Abramian et al. (19) destabilizes the rivers into several smaller channels that form a braided river. Curiously, this happens when the transport width of a river becomes comparable to its size. In the future, this may help us identify a mechanism for braiding, which is still debated $(3,6,34)$.

Our model provides a link between the shape of the river and its sediment load. It, thus, presents an opportunity for field measurements, whereby one could estimate the sediment discharge of a river by measuring its width and depth. Before this method can bed applied to natural rivers reliably, we should first extend the present theory to the case of turbulent flow, which will be the focus of future work.

ACKNOWLEDGMENTS. PP was supported by the JSMF postdoctoral fellowship. OD was partially funded by the "Emergence en Recherche" grant of the Université de Paris.

1. Parker $G$ (1978) Self-formed straight rivers with equilibrium banks and mobile bed. part 2. the gravel river. Journal of Fluid mechanics 89(1):127-146.

2. Schumm SA (1979) Geomorphic thresholds: the concept and its applications. Transactions of the Institute of British Geographers pp. 485-515.

3. Seminara G (2010) Fluvial sedimentary patterns. Annual Review of Fluid Mechanics 42:4366.

4. Métivier F, Barrier L (2012) Alluvial landscape evolution: What do we know about metamorphosis of gravel-bed meandering and braided streams?

5. Phillips CB, Jerolmack DJ (2016) Self-organization of river channels as a critical filter on climate signals. Science 352(6286):694-697.

6. Parker $\mathrm{G}$ (1976) On the cause and characteristic scales of meandering and braiding in rivers Journal of fluid mechanics 76(3):457-480.

7. Glover RE, Florey Q (1951) Stable channel profiles.

8. Henderson FM (1961) Stability of alluvial channels. Journal of the Hydraulics Division 87(6):109-138.

9. Parker G, Wilcock PR, Paola C, Dietrich WE, Pitlick J (2007) Physical basis for quasi-universa relations describing bankfull hydraulic geometry of single-thread gravel bed rivers. Journal of Geophysical Research: Earth Surface 112(F4).

10. Savenije HH (2003) The width of a bankfull channel; lacey's formula explained. Journal of Hydrology 276(1-4):176-183.

11. Métivier F, Lajeunesse E, Devauchelle O (2017) Laboratory rivers: Lacey's law, threshold theory, and channel stability.

12. Phillips C, Jerolmack DJ (2019) Bankfull transport capacity and the threshold of motion in coarse-grained rivers. Water Resources Research 55(12):11316-11330.

13. Malverti L, Lajeunesse E, Métivier F (2008) Small is beautiful: Upscaling from microscale laminar to natural turbulent rivers. Journal of Geophysical Research: Earth Surface 113(F4).

14. Ikeda S (1981) Self-formed straight channels in sandy beds. Journal of the hydraulics division 107(4):389-406.

15. Schumm SA, Mosley MP, Weaver W (1987) Experimental fluvial geomorphology

16. Federici B, Paola C (2003) Dynamics of channel bifurcations in noncohesive sediments. Water Resources Research 39(6).

17. Seizilles G, Devauchelle O, Lajeunesse E, Métivier F (2013) Width of laminar laboratory rivers. Physical Review E 87(5):052204.

18. Abramian A (2018) Ph.D. thesis.

19. Abramian A, Devauchelle O, Lajeunesse E (2020) Laboratory rivers adjust their shape to sediment transport. Physical Review E 102(5):053101.

20. Bagnold RA (1973) The nature of saltation and of ?bed-load?transport in water. Proceedings of the Royal Society of London. A. Mathematical and Physical Sciences 332(1591):473-504.

21. Seizilles G, Lajeunesse E, Devauchelle O, Bak M (2014) Cross-stream diffusion in bedload transport. Physics of Fluids 26(1):013302.

22. Abramian A, Devauchelle O, Seizilles G, Lajeunesse E (2019) Boltzmann distribution of sediment transport. Physical review letters 123(1):014501.

23. Parker G (1978) Self-formed straight rivers with equilibrium banks and mobile bed. part 1. the sand-silt river. Journal of Fluid Mechanics 89(1):109-125.

24. Shields A (1936) Application of similarity principles and turbulence research to bed-load movement.

25. Houssais M, Ortiz CP, Durian DJ, Jerolmack DJ (2015) Onset of sediment transport is a continuous transition driven by fluid shear and granular creep. Nature communications $6(1): 1-$

26. Pähtz T, Clark AH, Valyrakis M, Durán O (2020) The physics of sediment transport initiation, cessation, and entrainment across aeolian and fluvial environments. Reviews of Geophysics 58(1):e2019RG000679.

27. Salevan J, Clark AH, Shattuck MD, O'Hern CS, Ouellette NT (2017) Determining the onset of hydrodynamic erosion in turbulent flow. Physical Review Fluids 2(11):114302.

28. Lobkovsky AE, Orpe AV, Molloy R, Kudrolli A, Rothman DH (2008) Erosion of a granular bed driven by laminar fluid flow. Journal of Fluid Mechanics 605:47.

29. Charru F, Mouilleron $\mathrm{H}$, Eiff $\mathrm{O}$ (2004) Erosion and deposition of particles on a bed sheared by a viscous flow. Journal of Fluid Mechanics 519:55-80.

30. Charru $F(2006)$ Selection of the ripple length on a granular bed sheared by a liquid flow. Physics of fluids 18(12):121508.

31. Goodwin R, Homsy G (1991) Viscous flow down a slope in the vicinity of a contact line. Physics of Fluids A: Fluid Dynamics 3(4):515-528.

32. Andrews ED (1984) Bed-material entrainment and hydraulic geometry of gravel-bed rivers in colorado. Geological Society of America Bulletin 95(3):371-378.

33. Dunne KB, Jerolmack DJ (2020) What sets river width? Science advances 6(41):eabc1505

34. Leopold LB, Wolman MG (1957) River channel patterns: braided, meandering, and straight. (US Government Printing Office). 